\title{
Creep and Yield Loci of Zircaloy Cladding at Elevated Temperatures
}

\author{
K. LINGA MURTY, RATNAJI R. KOLA and SHEIKH T. \\ MAHMOOD
}

North Carolina State University, Raleigh, NC 27695-7909, USA

(Received 14 February 1986; in final form 20 March 1987)

Crystal plastic models are applied to textured Zircaloy in order to explain the recent experimental results on yield and creep loci by Suzuki et al. Crystallite orientation distribution functions were developed using texture pole figures obtained on cold-worked stress-relieved and recrystallized Zircaloy-4 tubings. The model predictions are in good agreement with experimental loci, and clearly point out the predominance of prism slip contribution for recrystallized material while the contribution of the basal slip increased as the amount of cold work increased.

KEY WORDS: Zircaloy, yield and creep loci, prism slip contribution.

Zirconium alloys are commonly used as thin-walled tubing in light water reactors, pressure tubes in steam generating heavy water reactors and calandria tubes in Canadian pressurized heavy water reactors. Zircaloy is the trade name for zirconium alloyed with tin and iron and has an hcp structure at service temperatures and transforms to bcc at about $1135 \mathrm{~K}$. In service, these cladding tubes are subjected to complex multiaxial loading due principally to the external pressure of the coolant and the internal pressure due to released fission gases. After sufficient burn-up, the pellet cladding mechanical interaction [PCI] adds to the complexity along with flow-induced vibration and spacer grid forces due to differential growth leading to fuel rod bow. In general, these tubes are thin-walled with diameter to thickness ratios of about 10 thereby 
leading to negligible radial [or thickness] stresses. The mechanical properties of hexagonal close packed [hcp] metals such as Zircaloy are sensitive to fabrication variables and the resulting crystallographic textures [Beauregard et al., 1977, Kallstrom, 1972, Tenckhoff, 1982]. The limited number of slip systems along with the inherent anisotropy of hexagonal crystal structure renders these materials highly textured with nonrandomly oriented grains. These preferred orientations are responsible for the resulting mechanical anisotropy of Zircaloy. Thus, recent emphasis has been on the behavior of the cladding subjected to biaxial loading [Murty and Adams, 1985, Kallstrom et al., 1974, Stehle et al., 1977, Baty et al., 1984, Murty and Yoon, 1979, Suzuki et al., 1985]. Suzuki et al., 1985 investigated the yield loci of commercial Zircaloy- 2 and Zircaloy- 4 cladding tubes at elevated temperatures by performing internal pressurization tests with superimposed axial loading. The goal of the present study has been to use the crystallographic texture combined with crystal plastic models to predict the yield loci of the textured polycrystal via crystallite orientation distribution functions [Bunge, 1982, Adams and Murty, 1985]. Suzuki et al., 1985 used recrystallized Zircaloy-2 and stress relieved Zircaloy-4. Since Suzuki et al. did not give results on crystallographic textures of their materials, we have considered the texture data on stressrelief annealed and recrystallized Zircaloy- 4 to generate the orientation distribution functions. It is believed that these texture data are representative of the typical cold-worked stress-relieved and recrystallized Zircaloys. We describe here the experimental results of Suzuki et al. on yield loci of Zircaloy-2, the crystallite orientation distribution functions derived from texture pole figures obtained on Zircaloy-4 and uniform-stress lower-bound analysis in predicting the yield loci of the textured cladding. The model predictions are in good agreement with experimental yield loci and clearly exhibit the predominance of prism slip contribution for recrystallized Zircaloy while the contribution of the basal slip becomes relatively more significant as the amount of cold-work increases.

\section{YIELD LOCI OF ZIRCALOY MATERIALS}

The materials used for obtaining the mechanical property data were recrystallized Zircaloy-2 and stress-relieved Zircaloy-4, typical com- 
Table 1 Compositions of zircaloys

\begin{tabular}{|c|c|c|c|c|c|c|}
\hline \multirow[b]{2}{*}{ Alloy } & \multicolumn{6}{|c|}{$\begin{array}{l}\text { Elements } \\
(w / 0)\end{array}$} \\
\hline & Sn & $\mathrm{Fe}$ & $\mathrm{Cr}$ & $\mathrm{Ni}$ & 0 & $\mathrm{Zr}$ \\
\hline Zircaloy-2 & 1.5 & 0.14 & 0.09 & 0.05 & 0.01 & balance \\
\hline Zircaloy-4 & 1.3 & 0.22 & 0.10 & - & 0.01 & balance \\
\hline
\end{tabular}

positions of which are given in Table 1. Suzuki et al., 1985 performed biaxial loading tests at elevated temperatures using these Zircaloy cladding tubes by subjecting them to axial tensile load and internal pressure simultaneously while maintaining the ratio of the hoop stress to axial stress $\left[\alpha=\sigma_{\theta} / \sigma_{z}\right]$ constant. They have performed constant stress ratio plastic deformation tests at different temperatures from which the yield loci were constructed at both $0.2 \%$ and $0.5 \%$ equivalent plastic strains $[\varepsilon]$. Figure 1 depicts the yield loci data at constant equivalent plastic strain of $0.5 \%$. In addition, Suzuki et al., 1985 performed creep tests at varied stresses

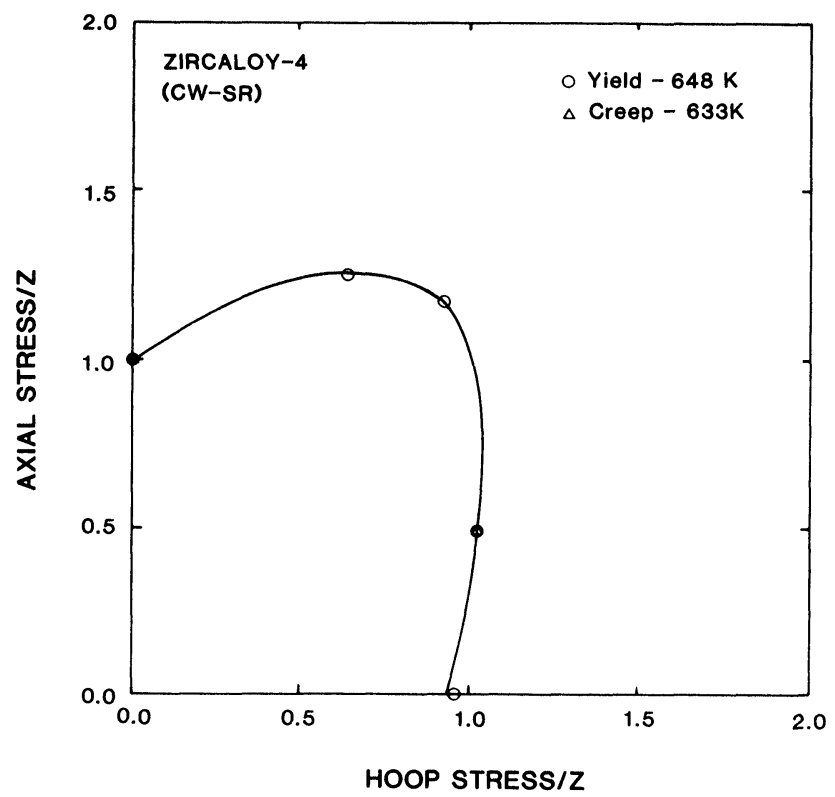

Figure 1a Yield loci of cold-worked stress-relieved Zircaloy-4 at $648 \mathrm{~K}$. Data obtained from creep potential at $633 \mathrm{~K}$ are also shown (Ref. 9). 


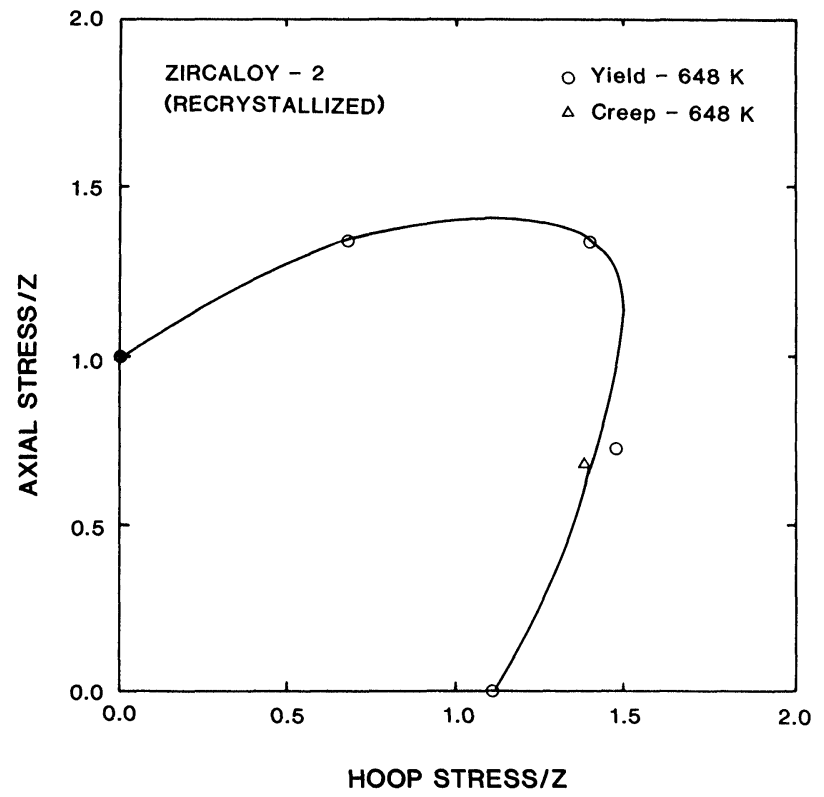

Figure 1b Yield loci of recrystallized Zircaloy-2 at $648 \mathrm{~K}$. Data obtained from creep potential are also shown (Ref. 9).

under biaxial closed end internal pressurization $[\alpha=2]$ and uniaxial $[\alpha=0]$ loadings. The creep loci were obtained at constant creep potential $[W]$ :

$$
W=\sigma_{\theta} \dot{\varepsilon}_{\theta}+\sigma_{z} \dot{\varepsilon}_{z},
$$

and these creep results are also shown in Figure 1. For comparison purposes, the loci results are normalized to the uniaxial stress along the axial direction $[Z]$. As to be noted in Figure 1 and as pointed out by Suzuki et al., anisotropy of creep and plastic deformation is identical. It should be noted that the deformation tests were performed at a constant strain-rate of $8.3 \times 10^{-6}$ per sec which is comparatively low and correspond to creep conditions. As pointed out by Beauregard et al., 1977 and Murty et al., 1982, mechanical anisotropy of Zircaloys is quite different under creep [low stress/high temperature] and tensile [high stress/low temperature] conditions which is attributed to changes in the deformation mode 
from high to low strain-rates [or low temperatures to high temperatures]. While Suzuki et al. reported data at various temperatures, we consider here their highest temperature results [648 K] where the power-law slip is known to be applicable [Murty and McDonald, 1982]:

$$
\dot{\varepsilon}=A \sigma^{n}
$$

\section{CRYSTALLOGRAPHIC TEXTURES OF ZIRCALOY-4}

Crystallographic texture pole-figures are commonly employed in the description of preferred orientations. Each pole-figure represents the spatial distribution of a given crystallographic plane within the polycrystal as portrayed in stereographic coordinates. It is the distribution of the measured diffracted X-ray intensity in space of a particular crystallographic plane. Particular planes are selected by fixing the Bragg angle. The reciprocal lattice vector [i.e., the diffraction direction] may be rotated with two degrees of freedom with respect to any arbitrary set of processing axes. The crystallographic pole figure is plotted as a stereographic projection showing times-random iso-intensity contours of the intensity distribution referred to the processing axes. In the present work, we employed the technique by Kula-Lopata (1962) to determine the texture pole figures. In this method the sample surface forms an angle of $54^{\circ} 44^{\prime}$ with each of the tube directions [radial, circumferential and axial]. While the advantage of this method lies in the representation of the stereographic projection without serious geometric defocussing problems, this involves a rather tedious specimen preparation by laminating flattened sections of the tube wall [without a thickness reduction] and slicing the laminate at the specified angle. The main advantage of this method is that it covers the full first quadrant $\left[0^{\circ}\right.$ to $90^{\circ}$ from the normal direction] and the assumption of four-fold symmetry allows extrapolation into the remaining three quadrants. This assumption is valid since all our Zircaloy pole figures show an orthorhombic statistical distribution of crystallites even though this is not required $a$ priori by the tube reduction processes. The added advantage of the Kula-Lopata method lies in the fact that one specimen is sufficient to generate the required pole figures. Basal (0001), prism (1010) and pyramidal 
(1012) plane pole figures were evaluated on cold-worked stressrelieved [cw-sr] and recrystallized Zircaloy-4 tubings. Figure 2 is a compilation of these pole-figures where the numbers on the iso-intensity contours indicate the times-random values. We note here the commonly observed bimodal distribution of the basal poles concentrated in the transverse plane normal to the tube axis $[r-\theta$ plane] with intensity maxima at $\pm 30^{\circ}-45^{\circ}$ from the radial direction towards the hoop [diametral]. The basal [0001] pole texture angle $\hat{\boldsymbol{\Phi}}$ [the angle between the radial direction and the $c$-axis of the average crystal in the $r-\theta$ plane] is sensitive to the strain path or cold work prior to the final annealing; the final annealing temperature has at best a minor effect on the basal pole angle and increases the population of randomly oriented crystallites resulting in a shallow maximum at $\hat{\boldsymbol{\Phi}}$. However, the final annealing temperature significantly affects the prism [1010] pole distribution as illustrated in Figure 2; the major texture modification due to recrystallization involves the rotation of the basal plane about the $c$-axis. Thus there exists a relatively high population of prism poles along the axial direction in $\mathrm{cw}-\mathrm{sr}$ while the population at $\pm 30^{\circ}$ from the axial direction increases following recrystallization anneal with essentially

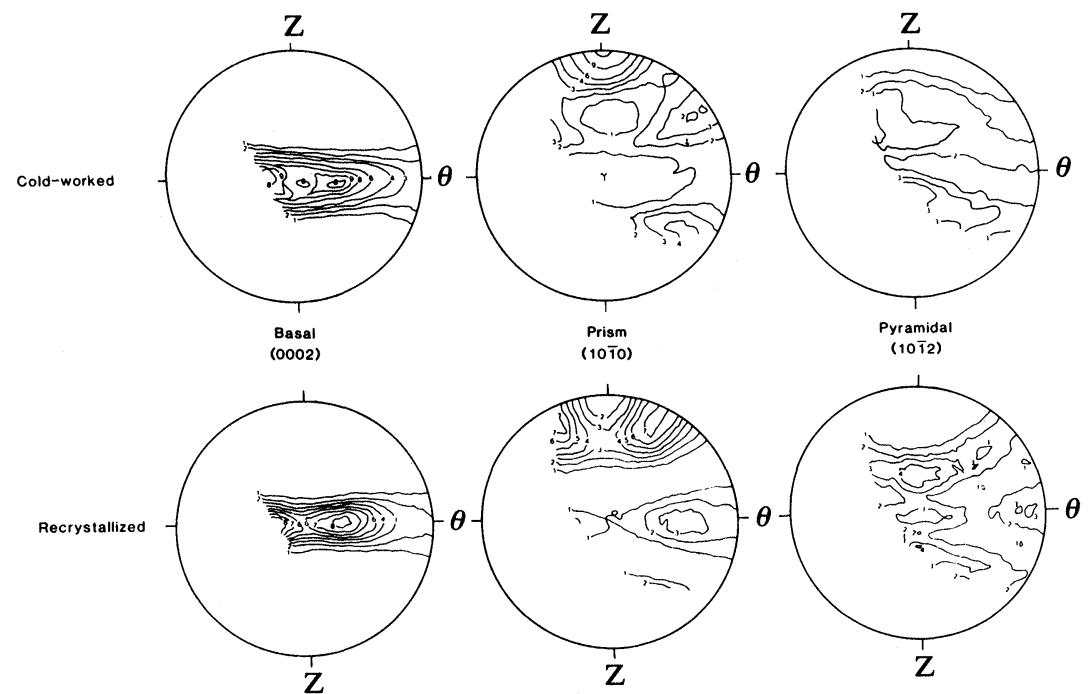

Figure 2 Crystallographic texture pole figures for cold-worked and recrystallized Zircaloy-4. 
no first order (1010) prism poles along the tube axial direction. As expected, the pyramidal pole distributions are relatively more random with peak intensities rarely exceeding 3-4 times the random in both the $\mathrm{cw}-\mathrm{sr}$ and recrystallized materials.

\section{THE CRYSTALLITE ORIENTATION DISTRIBUTION FUNCTION}

There is a serious drawback in the pole figure representation of preferred orientations since two or more pole figures are needed to qualitatively spot certain strong preferred orientations, the process which is not quantitative in nature and is often misleading. Quantitative representation of the textures is possible through an evaluation of a distribution function which gives the probability that a crystallite lies within certain range of orientations with respect to the specified macroscopic coordinates [generally processing axes]. This function, called the orientation distribution function $\omega(\theta, \psi, \phi)$, expresses the probability that a crystallite has an orientation $(\theta, \psi, \phi)$ with respect to the specimen axes. The CODF was defined in the space of Euler angles using the series method of Bunge (1965) and Roe (1965),

$$
\omega(\theta, \psi, \phi)=\sum_{l=0}^{\infty} \sum_{m=-l}^{l} \sum_{n=-l}^{l} W_{l m n} Z_{l m n}(\cos \theta) e^{-i m \psi} e^{-i n \phi},
$$

where $W_{l m n}$ are the appropriate series coefficients. In this Roe's formalism, $Z_{l m n}$ are the augmented Jacobi polynomials and $(\theta, \psi, \phi)$ are Euler angles connecting crystal coordinates [0-XYZ] with axes coincident with specimen and the principal working directions of the material $[0-x y z]$. In the present work, $Z$ was chosen to coincide with the $c$-axis of the crystallite, and $X$ and $Y$ are selected to be coincident with diagonal type-I [1120] and type-II [1010] axes respectively. The numerical coefficients $W_{l m n}$ are complex in general but they are real for cubic and hexagonal polycrystals exhibiting orthorhombic symmetry. Morris and Hekkler (1969) have shown that for centro and orthorhombic symmetry cases, $l$ and $m$ are restricted to even values and for hexagonal symmetry, $n$ is restricted to multiples of 6 in the expansion of Eq. (3). The restriction on $l$ is a result of the centrosymmetry inherent in the diffraction experiment and is not strictly demanded in the true 
orientation distribution function. The even part of the CODF $[l=0,2,4, \ldots]$ is deemed sufficient for the purposes of interest in evaluating yield loci, and this is the part which is readily determinable from the $x$-ray pole figure data. Neglecting the odd coefficients lead to negative values in the CODF which were simply omitted in the present analyses. With the aforementioned symmetry restrictions on $l, m$ and $n$, three pole figures [(0001), (1010), and (1012)] fixed a truncation level of $l$ equal to 16 .

The orientation distribution functions for cold-worked stressrelieved and recrystallized materials were generated using the pole-figure data in Fig. 2. The derived ODFs are shown in Figures $3 \mathrm{a}$ and $3 \mathrm{~b}$, respectively, in the form of Euler plots at constant intervals of $\phi$. The iso-times-random intensity contours are at constant intervals, and the contours corresponding to unity [random value] are noted in each of the slices in Figures $3 a$ and $3 b$. The shift of the prism pole distribution that occurred during annealing [Figure 2] is even more dramatically portrayed in the Euler plots. The peak times random value decreased slightly following recrystallization anneal; however, this is not as dramatic as noted earlier (Adams and Murty, 1985). Inherent in the series method of representation is the occurrence of 'negative' regions in the Euler

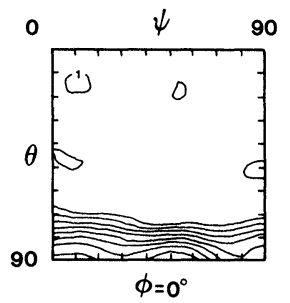

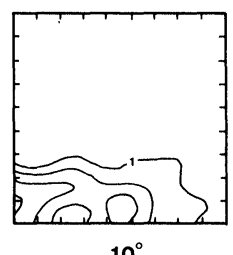
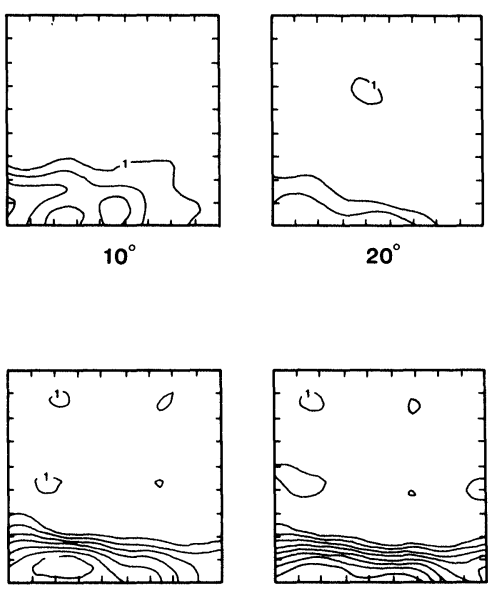

$50^{\circ}$ $20^{\circ}$

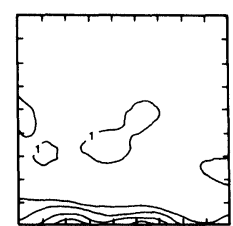

$30^{\circ}$

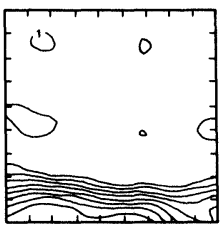

$60^{\circ}$

Figure 3a Euler plot representing ODF for cw-sr Zircaloy-4. 


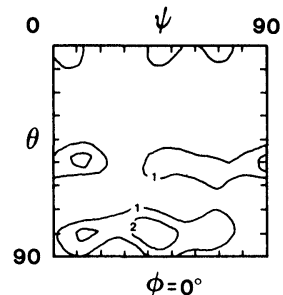

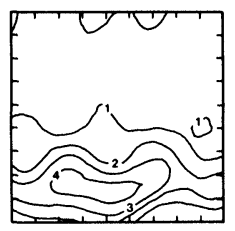

$10^{\circ}$

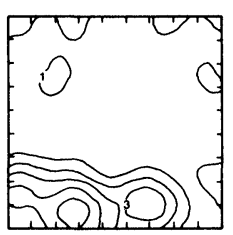

$40^{\circ}$

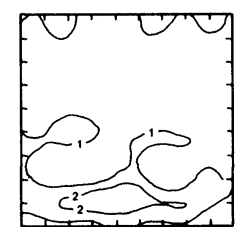

$50^{\circ}$

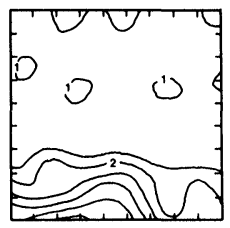

$20^{\circ}$

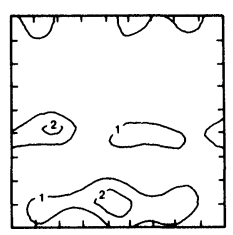

$60^{\circ}$

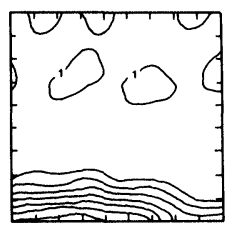

$30^{\circ}$

Recrystallized Zircaloy

Figure 3b Euler plot representing ODF for recrystallized Zircaloy-4.

plots and we observed negative regions of the order of $15 \%$. In depicting the ODFs as Euler plots, these negative regions are simply omitted.

As pointed out earlier, once the CODF is developed for a set of crystallographic texture pole figures, any orientation dependent property of a bulk polycrystal can be evaluated from single crystal data. Thus,

$$
\langle\rho(\theta, \psi, \phi)\rangle=\int_{0}^{2 \pi} \int_{0}^{2 \pi} \int_{-1}^{1} \rho(\theta, \psi, \phi) \omega(\theta, \psi, \phi) d(\cos \theta) d \psi d \phi
$$

where $\rho(\theta, \psi, \phi)$ is the microscopic crystal property and $\langle\rho(\theta, \psi, \phi)\rangle$ the corresponding property of the polycrystalline aggregate. Here, we assume that the contribution of grain-to-grain interactions is negligible and thus it is deemed sufficient to weigh the property by the CODF in evaluating the bulk behavior.

\section{UNIFORM STRESS POWER-LAW CREEP ANALYSIS}

The crystallite behavior in each orientation is weighted by the volume fraction of crystallites found in that orientation, namely CODF. In evaluating the polycrystalline yield behavior, rigid body 
models such as Sachs, Taylor and Bishop-Hill are commonly used where specific slip systems are postulated to operate (Adams and Murty, 1985, Adams, 1979). These models are applicable at relatively low temperatures where the plastic deformation is strainrate insensitive. The uniform stress lower-bound analysis, adopted here, satisfies stress equilibrium conditions but does not preserve the strain compatibility among the crystallites. All of the slip systems are considered to operate simultaneously in this model; however, the contribution of any given system is emphasized by an appropriate choice of the reference stresses. This analysis involves less computational time than the uniform strain upperbound scheme but this is commonly employed in the creep literature; moreover, it has been shown that for Zircaloys, both of these schemes yield results very close to each other (Adams and Murty, 1983). Power-law creep has been shown to be applicable for Zircaloys at the temperature and strain-rates used in evaluating the yield loci (Murty and McDonald, 1981). If $n$ is the stress exponent, the shear strain rate on the $s$ th slip system can be expressed as

$$
\dot{\gamma}^{(s)}=A\left|\frac{\tau^{(s)}}{\tau_{0}^{(s)}}\right|^{n}
$$

where $A$ is the reference strain rate, $\tau_{0}$ is the reference shear stress which is always positive and $n$ the stress exponent [=6]. The corresponding creep rate due to $s$ th slip system is given by

$$
\dot{\varepsilon}_{i j}^{(s)}=\mu_{i j}^{(s)} \dot{\gamma}^{(s)}
$$

The crystallite creep rate is then given by the sum of the contributions from the different slip systems.

$$
\dot{\varepsilon}_{i j}=\sum_{(s)} \mu_{i j}^{(s)} \dot{\gamma}^{(s)}
$$

When the resolved shear stress is equal to the reference stress, the strain rate becomes equal to the reference strain rate independent of the stress exponent. The shear stress is related to the crystallite stress $\left[\sigma_{i j}^{c}\right]$ using the fundamental tensor:

$$
\tau^{(s)}=\sigma_{i j}^{c} \mu_{i j}^{(s)},
$$

where the superscript $s$ refers to the $s$ th slip system. For isothermal modelling it is not possible to separate the reference strain rate $[A]$ and the reference shear stress and thus it is convenient to refer to a 
reference creep compliance $\dot{M}_{0}^{(s)}$ which is defined as

$$
\dot{M}_{0}^{(s)}=\frac{A}{\left[\tau_{0}^{(s)}\right]^{n}} .
$$

In the lower bounding model, the stress state in each crystallite $\left(\sigma_{i j}^{c}\right)$ is equated to the macroscopic stress state $\left(\sigma_{i j}\right)$, and the lower bound estimate of the strain-rate, $\dot{\varepsilon}_{i j}$ can be calculated from the orientation distribution function using the averaging process:

$$
\dot{\varepsilon}_{i j}=\left\langle\dot{\varepsilon}_{i j}^{c}\right\rangle=\int_{0}^{2 \pi} \int_{0}^{2 \pi} \int_{-1}^{1} \omega(\theta, \psi, \phi) \dot{\varepsilon}_{i j}^{c}(\theta, \psi, \phi) d(\cos \theta) d \psi d \phi
$$

where $\omega(\theta, \psi, \phi)$ is the distribution function with $(\theta, \psi, \phi)$ being the orientation of the crystallite. Thus, in the lower-bound estimate, stress equilibrium is artificially preserved at the expense of violating strain rate compatibility.

In the present analysis, three different slip modes are considered: basal $(\{0001\}\langle 11 \overline{2} 0\rangle)$, prismatic $(\{10 \overline{1} 0\}\langle 11 \overline{2} 0\rangle)$ and pyramidal $(\{11 \overline{2} 2\}\langle 11 \overline{2} 3\rangle)$ with the respective reference stresses denoted as $\tau_{B}, \tau_{A}, \tau_{p}$. Values of the reference stresses are chosen to emphasize the contribution of individual or particular combinations of slip modes to the polycrystalline behavior. To evaluate the contribution of prismatic slip, for example, the reference stresses $\tau_{B}$ and $\tau_{P}$ are taken as 1000 times $\tau_{A}$.

Before correlating the model predictions with the experimental results, they need to be normalized to reflect changes in the rate $W$ of creep dissipation and the reference creep compliance $\dot{M}_{0}^{(s)}$. Let the imposed stress state be altered by a scale factor such that

$$
\sigma_{i j}^{*}=\lambda \sigma_{i j} \text {. }
$$

In the present analyses the reference creep compliance is assumed to be constant $\dot{M}_{0}$, i.e., independent of the slip system. The rate of creep dissipation can then be written for the new stress state as,

$$
\begin{aligned}
W^{*}= & \lambda \sigma_{i j} \dot{M}_{0} \sum_{(s)} \mu_{i j}^{(s)}(\bar{g})\left|\mu_{p q}^{(s)}(\bar{g}) \lambda \sigma_{p q}\right|^{n} \\
& \times \operatorname{sgn}\left\{\mu_{p q}^{(s)}(\bar{g}) \lambda \sigma_{p q}\right\} \\
= & \lambda|\lambda|^{n} \operatorname{sgn}(\lambda) \sigma_{i j} \dot{M}_{0} \sum_{(s)} \mu_{i j}^{(s)}(\bar{g}) \\
& \times\left|\mu_{p q}^{(s)}(\bar{g}) \sigma_{p q}\right|^{n} \operatorname{sgn}\left\{\mu_{p q}^{(s)}(\bar{g}) \sigma_{p q}\right\} \\
= & |\lambda|^{n+1} W
\end{aligned}
$$


where $\bar{g}$ symbolizes crystal orientation $\theta \psi \phi$. Thus the scaled stress state becomes

$$
\sigma_{i j}^{\prime}=\left(\frac{W^{*}}{W}\right)^{1 /(n+1)} \operatorname{sgn}(\lambda) \sigma_{i j},
$$

accounting for the changes in the rate of creep dissipation. This equation enables the adjustment of the scale for yield and creep loci determinations. Also, this equation can be used to find values for the reference creep compliance consistent with measured experimental values. The above equation implies that increasing the stress by a factor of $\lambda$ does not alter the orientation-dependent distribution of strain-rates; the strain-rates are simply scaled.

\section{RESULTS AND DISCUSSION}

As pointed out earlier, the orientation distribution function was generated from the two sets of the three pole figures in Figure 2. A stress exponent of 6 for $n$ in Eq. (5) is used in these analyses and 486 texture components are considered to numerically evaluate Eq. (8). Details on the computational scheme and computer algorithm may be found in Adams, 1979. In obtaining the loci, the rate $W$ of creep dissipation was fixed at a constant value using the scaling laws presented in the earlier section and the stresses were normalized to the uniaxial stress $[Z]$. This allows a comparison of the predictions of the different slip systems with the experimental data. While we have scaled the model predictions to a constant $W[8.33 \times$ $10^{-6} \mathrm{MPa} / \mathrm{s}$, we assumed that the creep compliance $\dot{M}_{0}^{(s)}$ is identical for all slip systems which may not necessarily be true. There is no method of evaluating these compliances for a given slip system at the present time. Earlier investigations on lower-bound and upperbound estimates of this sort revealed that this assumption is quite valid. Figure 4 depicts the normalized loci obtained from the model based on easy slip on basal, prismatic and pyramidal slip systems using recrystallized texture along with the experimental data from deformation and creep tests at $648 \mathrm{~K}$. The results show an excellent agreement between the experimental data on recrystallized Zircaloy-2 and model predictions based on dominant slip on prism 


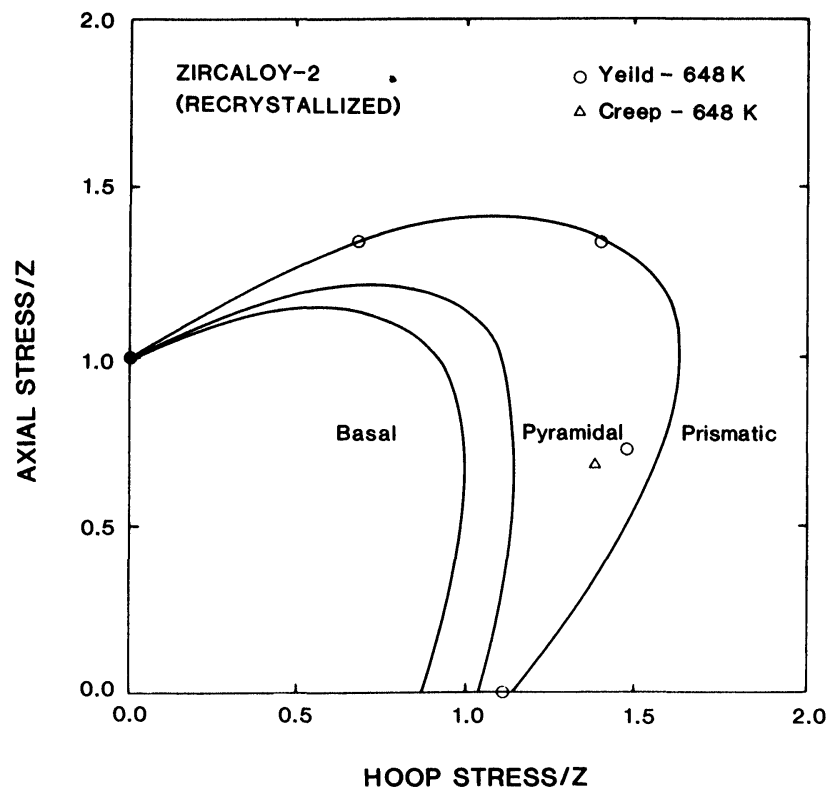

Figure 4 Comparison between the experimental yield and creep loci data for recrystallized Zircaloy- 2 and model predictions based on contributions from basal, prismatic and pyramidal slip, respectively, using texture data of recrystallized Zircaloy-4.

planes. This is in agreement with single crystal slip measurements which indicate that $\{10 \overline{1} 0\}\langle 11 \overline{2} 0\rangle$ type prismatic slip dominates in low $c / a$ ratio hexagonal close packed materials. Slight deviations of the experimental data from model predictions are believed to be due primarily to the contribution of pyramidal slip to the overall deformation behavior. Moreover, the scaling of the compliance might have a minute influence in obtaining a better correlation between the model predictions and the experimental data. Considering these factors, the correlation between the experimental data and model predictions is regarded very good.

Similar correlations are shown in Figure 5 for the cold-worked stress-relieved Zircaloy- 4 and model predictions were made using the $\mathrm{cw}-\mathrm{sr}$ texture [Figure 2]. The primary difference between the loci of $\mathrm{cW}-\mathrm{sr}$ and recrystallized materials lies in the fact that cold working resulted in substantial hardening for stress states below 
equibiaxial $[\alpha=1]$ loading while recrystallized materials exhibit loci stretched to higher hoop stresses for the stress ratios equal to or larger than unity. Correlations clearly indicate the importance of pyramidal and basal slip for stress ratios larger than unity while prism slip dominant deformation behavior occurs at lower $\alpha$ values. Although the experimental results do not correlate with the basal slip predictions, the general shapes of the experimental and predicted loci are very similar. The poor correlation in the $\mathrm{cw}-\mathrm{sr}$ case is believed to be due to the fact that the crystallographic textures are very sensitive to the amount of cold-work and the degree of stress relief and the materials [although Zircaloy-4 in both cases] used in obtaining the texture data are different from those used in deformation tests. It is interesting to note that the earlier work (Murty and Adams, 1982) indicated the dominance of basal slip on the creep loci of cw-sr Zircaloy-4. It is clear from Figures 4 and 5 that the contribution of prism slip to the overall deformation of Zircaloys decreases as the amount of cold work increases. This is explained on the basis that the cold work during fabrication of these cladding tubes might have induced a large amount of dislocations on the principal slip system, namely the prismatic planes [in low $c / a$ ratio materials] thereby resulting in increased reference stress [Eq. (5)] for slip on prismatic planes. Second order slip systems such as the basal slip start operating during subsequent deformation of these cold worked [and partially stress relieved] Zircaloys. This is further inferred from the fact that recrystallization annealing treatment brought back the dominance [Figure 4] of prismatic slip by annealing the damage created during the fabrication process. The fact that pyramidal slip is much closer to the $\mathrm{cw}-\mathrm{sr}$ data is strongly corroborated by transmission electron microscopy observations which revealed a substantial $c$ component in cold-worked stress-relieved Zircaloy. Moreover, the contribution of pyramidal slip plays an important role of fulfilling the requirement of five independent slip systems for homogeneous plastic deformation. Thus some degree of pyramidal slip (or twinning) is expected in any compatible deformation model even though the reference shear stresses for this mode might be high.

We note from Figures 4 and 5 that the textural modification following recrystallization does not significantly affect the slip predictions. The differences in the deformation behaviors of the 


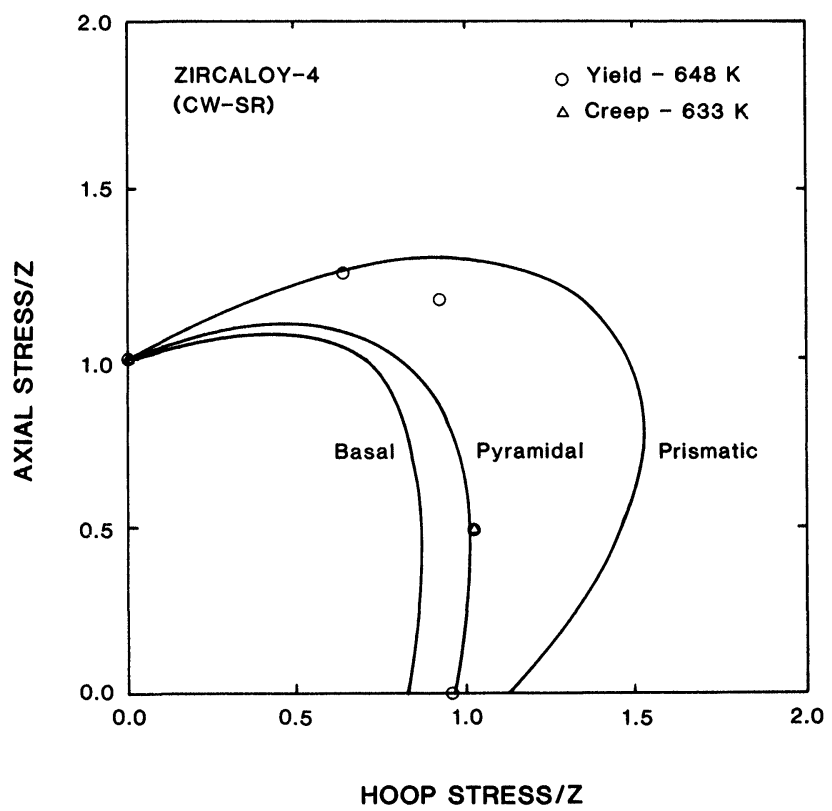

Figure 5 Comparison between the experimental yield and creep loci data for cold-worked stress-relieved Zircaloy-4 and model predictions based on contributions from basal, prismatic and pyramidal slip, respectively.

cw-sr and recrystallized Zircaloys are principally due to the changes in the operating slip modes rather than the texture per se. It should be emphasized that it is possible to fit the experimental data with simulations using combinations of the three slip modes with judiciously chosen sets of reference stresses. However, these predictions would be of limited use without further corroborative data from electron microscopic observations.

\section{Acknowledgments}

This work is supported by the National Science Foundation through the grant DMR 83-13157. Acknowledgements are due to Prof. B. L. Adams for assistance in the model development and many discussions. 


\section{References}

Adams, B. L., "An Upper-Bound Analysis of Inelastic Deformation in Textured Hexagonal Polycrystals (Zircaloy)," Ph.D. Thesis, The Ohio State University (1979).

Adams, B. L. and Murty, K. L., in Proceedings of the 4th International Conference on Mechanical Behavior of Materials, Stockholm, Pergamon Press (1983) 165.

Adams, B. L. and Murty, K. L., Mat. Sci. Eng., 70 (1985) 181.

Baty, D. L., et al., in 'Zirconium in the Nuclear Industry,' ASTM STP 824 (1984) 306.

Beauregard, R. J., Clevinger, G. S. and Murty, K. L., in Proceedings of the 4th International Conference on SMIRT, San Francisco (1977) paper C 3/5.

Bunge, H. J., Z. Metallk., 56 (1965) 872.

Bunge, H. J., 'Texture Analysis in Materials Sciences,' Butterworths (London) 1982.

Kallstrom, K., Can. Met. Q. 11 (1972) 185.

Kallstrom, K., Andersson, T. and Hofvenstam, A., in 'Zirconium in Nuclear Applications,' ASTM STP 551 (1974) 160.

Lopata, S. L. and Kula, E. B., Trans. AIME, 224 (1962) 865.

Morris, P. R. and Heckler, A. J., Trans. AIME, 245 (1969) 1877.

Murty, K. L. and Adams, B. L., in 'Mechanical Testing for Deformation Model Development,' ASTM STP 765 (1982) 382.

Murty, K. L. and Adams, B. L., Mat. Sci. Eng., 70 (1985) 169.

Murty, K. L. and McDonald, S. G., in Proceedings of 6th International Conference on SMIRT, Paris (1981) paper c $2 / 3$.

Murty, K. L. and Yoon, K. K., Scripta Met., 13 (1979) 299.

Roe, R. J., J. Appl. Phys., 37 (1965) 2024.

Stehle, H., Steinberg, E. and Tenckhoff, E., in 'Zirconium in Nuclear Industry,' ASTM STP 633 (1977) 486.

Suzuki, H., Okubo, T., Machida, K. and Kosugi, Y., "Determination of Yield Loci of Zircaloy Cladding Tubes at Elevated Temperature," presented at 7th International Conference on 'Zirconium in the Nuclear Industry,' 24-27 June 1985, Strassbourg, France, ASTM STP (in print).

Tenckhoff, E., in 'Zirconium in the Nuclear Industry,' ASTM STP 754 (1982) 5. 